\title{
O uso de medicamentos na sociedade que envelhece: uma revisão literária
}

\author{
The use of medications in an aging society: a literary review \\ El uso de medicamentos en una sociedad que envejece: una revisión literaria
}

Recebido: 07/11/2021 | Revisado: 15/11/2021 | Aceito: 23/11/2021 | Publicado: 03/12/2021

\author{
Bianca Jenifer de Sá da Silva \\ ORCID: https://orcid.org/0000-0002-5962-7348 \\ Faculdade de Palmas, Brasil \\ E-mail: bianccadesa@gmail.com \\ Márcio Trevisan \\ ORCID: https://orcid.org/0000-0002-7597-5481 \\ Faculdade de Palmas, Brasil \\ E-mail: marciotrevi@mail.uft.edu.br
}

\begin{abstract}
Resumo
A sociedade que envelhece tem mais predisposição a ter doenças e/ou dores em decorrência das limitações causadas pelo envelhecimento. A solução mais rápida e de fácil acesso para retardar os sintomas de doenças e aliviar dores são o uso de medicamentos. Este trabalho tem por objetivo caracterizar o uso de medicamentos por idosos, analisar os fatores que influenciam neste processo e identificar as consequências do uso de medicamentos pela terceira idade, por meio de revisão de literatura de abordagem qualitativa. Os resultados obtidos foram o hábito da automedicação, o descarte indevido dos fármacos e a prática da polifarmácia, que são influenciados por propagandas assistidas na mídia, práticas populares e ineficiência do sistema público de saúde, que resultam em interações medicamentosas e reações adversas.

Palavras-chave: Medicamentos; Idoso; Polifarmácia; Automedicação; Evento adverso.
\end{abstract}

\begin{abstract}
The aging society is more likely to have diseases and/or pain as a result of the limitations caused by aging. The quickest and most easily accessible solution to delay disease symptoms and relieve pain is the use of medication. This study aims to characterize the use of medication by the elderly, analyze the factors that influence this process and identify the consequences of the use of medication by the elderly, through a literature review with a qualitative approach. The results obtained were the habit of self-medication, the improper disposal of drugs and the practice of polypharmacy, which are influenced by advertisements seen in the media, popular practices and inefficiency of the public health system, which result in drug interactions and adverse reactions.
\end{abstract}

Keywords: Medicines; Aged; Polypharmacy; Self-medication; Adverse event.

\section{Resumen}

La sociedad que envejece tiene más probabilidades de tener enfermedades y / o dolor como resultado de las limitaciones causadas por el envejecimiento. La solución más rápida y accesible para retrasar los síntomas de la enfermedad y aliviar el dolor es el uso de medicamentos. Este estudio tiene como objetivo caracterizar el uso de medicamentos por los ancianos, analizar los factores que influyen en este proceso e identificar las consecuencias del uso de medicamentos por los ancianos, a través de una revisión de la literatura con un enfoque cualitativo. Los resultados obtenidos fueron el hábito de la automedicación, la disposición inadecuada de medicamentos y la práctica de la polifarmacia, los cuales están influenciados por la publicidad vista en los medios, las prácticas populares y la ineficiencia del sistema de salud pública, que resultan en interacciones medicamentosas y reacciones adversas.

Palabras clave: Medicamentos; Anciano; Polifarmacia; Automedicación; Acontecimiento adverso.

\section{Introdução}

A terceira idade, composta por pessoas a partir dos 60 anos de acordo a Organização das Nações Unidas (ONU), é uma fase da vida que carrega limitações trazidas pelo corpo decorrentes da naturalidade da idade, de vícios, doenças, etc, que tornam o idoso mais frágil, além de questões financeiras, ingestão de vários medicamentos, reações adversas a medicamentos, que acentuam o problema (Flores \& Mengue, 2005).

Devido a ações de saúde pública implantadas ainda nas décadas de 40 a 70, houve um aumento da expectativa de vida no Brasil. Ações como vacinação e saneamento básico contribuíram para que a parcela de idosos no país aumentasse 
consideravelmente. As projeções são de que até 2050 o aumento de idosos no Brasil faça com que este se torne o sexto país com maior população idosa do mundo (Nóbrega \& Karnikowsky, 2005).

Os dados do Instituto Brasileiro de Geografia e Estatística (IBGE) de 2018 mostram que o país tinha 30,2 milhões de idosos em 2017. Do total, 16,9 milhões (56\%) são mulheres e 13,3 milhões (44\%) são homens. O número de homens é menor em decorrência do fato dos homens se cuidarem menos e não procurarem participar de programas de prevenção, como fazer exames de rotina (Vieiro et al., 2020).

A medida que o corpo envelhece, este passa a reagir de forma mais lenta e reduzida se comparada a outras idades. E tudo isso é a ordem natural da vida. O organismo deixa de trabalhar acelerado, a memória falha, os movimentos ficam mais lentos. E aí surgem alguns problemas. Esquecimento, falta de discernimento e doenças passam a gerar conflitos na vida de idosos (Vieiro et al., 2020).

O metabolismo desacelerado, confuso e/ou doente de um idoso requer o uso de medicamentos para controlar alguma doença. O processo de envelhecimento é marcado por uma elevação da frequência de doenças crônico-degenerativas, o que aumenta a demanda pelos serviços de saúde e por medicamentos, e expõe a população idosa aos riscos da prática de polifarmácia e a ocorrência mais frequente de efeitos adversos (Nóbrega \& Karnikowsky, 2005).

No Brasil, a criação do Sistema Único de Saúde (SUS) contribuiu para que os serviços de saúde chegassem até os idosos. O SUS é um sistema de saúde gratuito, que preza por um atendimento universal, integral e equitativo a todos. As demandas mais recebidas por este sistema são relativas a doenças crônicas não-transmissíveis, como hipertensão arterial e diabetes, condicionadas ao tabagismo, maus hábitos alimentares e falta de exercícios físicos (Vieiro et al., 2020).

Os idosos tendem a tomar mais medicamentos do que os jovens por serem mais predispostos a terem doenças. A maior parte dos medicamentos usados por eles para disfunções crônicas são tomados por anos (Vieiro et al., 2020). E nem sempre o idoso consegue gerenciar da forma correta o uso do medicamento da qual necessita.

A grande quantidade de medicações tomadas por idosos aumentam o número de problemas com medicamentos, o número de reações adversas e procura por serviços médico-hospitalares (Oliveira \& Novaes, 2012). Em contrapartida, a intervenção farmacológica é a medida mais aplicada para sanar problemas com idosos (Secoli, 2010).

O uso de medicamentos e a intercessão dos farmacêuticos são importantes cuidados para os idosos, pois possibilitam uma assistência farmacoterapêutica correta, de forma a evitar complicações. A intervenção educativa do profissional de farmácia é fundamental para uma boa qualidade de vida e criação de bons hábitos pelos idosos, visto que pertencem a um grupo mais vulnerável, que necessita de maior atenção, o que implica na necessidade de auxílio especializado para cuidado das especificidades de cada um (Melo \& Vale, 2018).

Consequentemente eles são mais suscetíveis aos efeitos adversos dos medicamentos por diversos fatores, como a utilização excessiva e muitas das vezes de forma incorreta, a função redutora de vários sistemas fisiológicos e o metabolismo alterado (Nóbrega \& Karnikowsy, 2005). Portanto, qualquer problema ou dúvida relacionado a um medicamento deve ser comunicado e conversado com um médico ou farmacêutico para evitar problemas e promover a boa saúde.

A intervenção pedagógica nestes casos tem grande relevância para o fortalecimento de hábitos saudáveis relacionados aos medicamentos. Assim, este trabalho tem por objetivo geral caracterizar o uso de medicamentos por idosos, e por objetivos específicos analisar, com base na literatura, os fatores que influenciam neste processo e identificar as consequências do uso de medicamentos.

\section{Metodologia}

Trata-se de uma revisão integrativa sobre o uso de medicamentos pela sociedade que envelhece, com base em artigos científicos selecionados nas bases de dados eletrônicas de busca Scientic Eletronic Library Online (SciELO), Literatura Latino- 
Americano e do Caribe em Ciências da Saúde (LILACS) e Medical Literature Analysis And Retrieval System Online (MEDLINE). Com base em Pereira et al. (2018), em uma revisão deve ser usado apenas autores que tenham relação com o assunto e participarão da discussão de fato. Para Souza, Silva \& Carvalho (2010), a revisão integrativa permite a análise de estudos experimentais e não-experimentais sobre um mesmo assunto para se chegar a um resultado. Busca a definição, revisão e análise de problemas.

Para sustentar a busca pelos artigos, foram utilizados palavras chaves e descritores como "uso de medicamentos", "idoso", "administração medicamentosa", "polifarmácia", "prescrição medicamentosa", checados junto ao DECs (Descritores em Ciências da Saúde) garantindo assim a utilização de termos relevantes para as referidas buscas.

As pesquisas foram conduzidas pelos autores de forma independente nos bancos de dados propostos, sendo que para maximizar e refinar a seleção, foi aplicado o cruzamento dos descritores entre si com auxílio do termo booleano "e/AND" e suas combinações utilizadas em português e inglês, favorecendo assim uma seleção refinada de artigos.

Inicialmente os artigos foram selecionados a partir da leitura título em português ou inglês e que revelara haver clara ligação com o tema deste trabalho. Estes artigos incialmente selecionados foram reclassificados obedecendo aos critérios de inclusão, de ter resumo disponível em português ou inglês, estar disponível na íntegra para leitura, ter sido publicado entre 2005 e 2021, possuir clara indicação de autoria, ter sido publicados em periódicos indexados nos referidos bancos de dados.

Foram considerados critérios de exclusão, não estar disponível na integra para leitura, terem data de publicação fora do período de 2005 a 2021, ou que após a leitura não estavam relacionados ao tema deste trabalho, conforme Figura 1.

Figura 1: Fluxograma de seleção de artigos para compor a revisão integrativa.

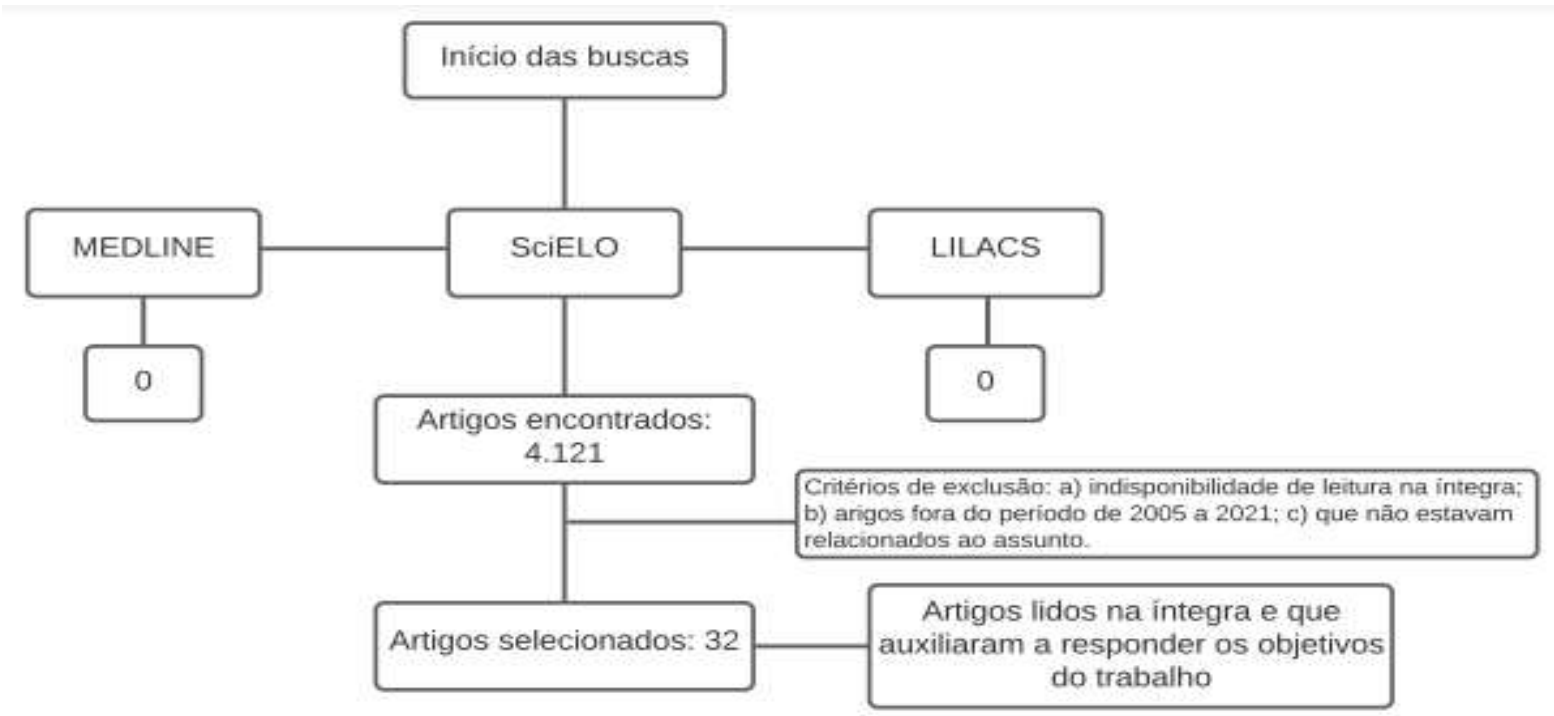

Fonte: Autores.

As informações extraídas dos estudos selecionados foram realizadas de forma descritiva e qualitativa, possibilitando assim, observar, contar descrever, classificar os resultados e argumentar sobre o tema explorado nessa revisão, de acordo os artigos do quadro resumo a seguir. 
Quadro 1: Identificação dos artigos selecionados.

\begin{tabular}{|c|c|c|c|}
\hline REVTSTA & ARTIGO & AUTORES & ANO \\
\hline Ciência \& Saúde Coletiva & $\begin{array}{l}\text { Análise do perfil de } \\
\text { automedicaçăo em mulheres } \\
\text { idosas brasileiras }\end{array}$ & $\begin{array}{l}\text { Bortolon, P., Medeiros, E., } \\
\text { Naves, J., Karnikowsky. } \\
\text { M., \& Nóbrega, O. }\end{array}$ & 2008 \\
\hline Revista Médica de Minas Gerais & $\begin{array}{l}\text { A prescriçāo de medicamentos } \\
\text { para idosos internados em serviço } \\
\text { de clínica médica: atualizaçāo. }\end{array}$ & $\begin{array}{l}\text { Costa, S. C. C., \& Pedroso, } \\
\text { E. R. P. }\end{array}$ & 2010 \\
\hline $\begin{array}{l}\text { In: Congresso Brasileiro de } \\
\text { Enfermagem, } 61^{\circ}\end{array}$ & $\begin{array}{l}\text { A influência da midia na } \\
\text { promoção da Saúde }\end{array}$ & $\begin{array}{l}\text { Dias, } F_{*} \text { Silva, } \mathrm{K} ., \text { Lima, } \\
\mathrm{F}_{\rightarrow,} \text { \& Pinheiro, } \mathrm{P} \text {. }\end{array}$ & 2009 \\
\hline Revista Saúde Pública & $\begin{array}{l}\text { Uso de medicamentos por idosos } \\
\text { em região sul do Brasil }\end{array}$ & $\begin{array}{l}\text { Flores, L. M. \& Mengue, } \\
\text { S. S. }\end{array}$ & 2005 \\
\hline Rev. CEFAC & $\begin{array}{l}\text { Saúde e } \\
\text { quilombolas: uma revisão da } \\
\text { literatura }\end{array}$ & $\begin{array}{l}\text { Freitas, D., Caballero, A., } \\
\text { Marques, A., Hernández, } \\
\text { C.. \& Antunes, S. }\end{array}$ & 2011 \\
\hline Santa Maria & $\begin{array}{l}\text { Interaçòes medicamentosas } \\
\text { potenciais na farmacoterapia de } \\
\text { idosos atendidos em farmácia } \\
\text { básica do sul do Brasil }\end{array}$ & $\begin{array}{l}\text { Garske, C., Assis, M., } \\
\text { Schneider, A., Machado, } \\
\text { E., \& Morsch, L. }\end{array}$ & 2016 \\
\hline $\begin{array}{l}\text { Rev. Bras. Med. Fam. } \\
\text { Comunidade, }\end{array}$ & $\begin{array}{l}\text { Prevalència e fatores associados a } \\
\text { potenciais interaçôes } \\
\text { medicamentosas entre idosos em } \\
\text { um estudo de base populacional }\end{array}$ & $\begin{array}{l}\text { Gotardelo, D., Fonseca, } \\
\text { L.. Masson, E., Lopes, L., } \\
\text { Toledo, V. Faioli, M., } \\
\text { Meira, A., Costa, C., \& } \\
\text { Andrade, R. }\end{array}$ & 2014 \\
\hline IBGE & $\begin{array}{l}\text { Número de idosos cresce } 18 \% \mathrm{em} \\
5 \text { anos e ultrapassa } 30 \text { milhỏes em } \\
2017\end{array}$ & - & 2018 \\
\hline IBGE & $\begin{array}{l}\text { População brasileira deve chegar } \\
\text { ao máximo } 228,4 \text { milhōes em } \\
2042\end{array}$ & - & 2013 \\
\hline Ciência \& Saúde Coletiva & $\begin{array}{l}\text { Influência da propaganda na } \\
\text { utilização de medicamentos em } \\
\text { um grupo de idosos atendidos em } \\
\text { uma unidade básica de saúde em } \\
\text { Aracajú (SE, BRASIL) }\end{array}$ & $\begin{array}{l}\text { Junior, D., Neves, A., } \\
\text { Cerqueira, C., Marcellini, } \\
\text { P., Marques, T., \& Barros, } \\
\text { J. }\end{array}$ & 2010 \\
\hline $\begin{array}{l}\text { Revista Amazônica Science \& } \\
\text { Health }\end{array}$ & $\begin{array}{l}\text { Polifarmácia: uso } \quad \text { de } \\
\text { medicamentos por idosos }\end{array}$ & Melo, L. M., \& Vale, B. N. & 2018 \\
\hline Ministério da Saúde & $\begin{array}{l}\text { Saúde da pessoa idosa: prevençào } \\
\text { e promoçăo à saúde integral }\end{array}$ & - & 2021 \\
\hline Ciência \& \& Saúde & $\begin{array}{l}\text { Propaganda de medicamentos } \\
\text { para grande público: parâmetros } \\
\text { conceituais de uma prática } \\
\text { produtora de risco }\end{array}$ & Nascimento, A. C. & 2010 \\
\hline Ciència \& Saúde Coletiva & $\begin{array}{l}\text { A terapia medicamentosa no } \\
\text { idoso: cuidados na medicação }\end{array}$ & $\begin{array}{l}\text { Nóbrega, O. T.. } \quad \text { \& } \\
\text { Karnikowsky, M. G. O. }\end{array}$ & 2005 \\
\hline Universidade Aberta do SUS & $\begin{array}{l}\text { Fatores de risco associados à } \\
\text { polifarmácia no idoso }\end{array}$ & Oliveira, A. M. & 2018 \\
\hline Saúde e Pesquisa & $\begin{array}{l}\text { Desenvolvimento, validaçào e } \\
\text { utilização de material educativo } \\
\text { sobre armazenamento correto de } \\
\text { medicamentos }\end{array}$ & $\begin{array}{l}\text { Oliveira, D., Jesus, P., } \\
\text { Zucco, B., Panosso, Ë., } \\
\text { Rocha, V., Bayer, V., \& } \\
\text { Ries, E. }\end{array}$ & 2020 \\
\hline
\end{tabular}




\begin{tabular}{|c|c|c|c|}
\hline Revista Brasileira de Enfermagem & $\begin{array}{l}\text { Uso de medicamentos por idosos } \\
\text { de instituiçōes de longa } \\
\text { permanência, Brasilia-DF, Brasil }\end{array}$ & $\begin{array}{l}\text { Oliveira, M. P. F., \& } \\
\text { Novaes, M. R. C. G. }\end{array}$ & 2012 \\
\hline UNIVILLE & $\begin{array}{l}\text { Riscos da } \quad \text { automedicação: } \\
\text { tratando o problema com } \\
\text { conhecimento }\end{array}$ & $\begin{array}{l}\text { Pereira, J., Soares, L., } \\
\text { Hoepfner, L., Kruger, K., } \\
\text { Guttervil, M., Tonini, K., } \\
\text { Devegili, D., Rocha, E., } \\
\text { Verdi, F., Dalfovo, V., } \\
\text { Oslen, K., Mendes, } \text { T., }_{\text {., }} \\
\text { Deretti, R. Soares, V., } \\
\text { Lobermeyer, C., Moreira, } \\
\text { J., Ferreira, J., \& } \\
\text { Francisco, A. }\end{array}$ & 2006 \\
\hline Revista Dor & $\begin{array}{l}\text { Prevaléncia da dor e do uso de } \\
\text { analgésicos e antiinflamatórios na } \\
\text { automedicaçào de pacientes } \\
\text { atendidos no Pronto-Socorro } \\
\text { Municipal de Taubaté }\end{array}$ & $\begin{array}{l}\text { Piotto, F., Nogueira, R., } \\
\text { Pires, O., et al. }\end{array}$ & 2009 \\
\hline BRASIL & $\begin{array}{l}\text { Resolução da Diretoria Colegiada } \\
n^{\circ} 222\end{array}$ & - & 2018 \\
\hline Universidade de Brasilia & $\begin{array}{l}\text { Educação em saúde para a } \\
\text { promoção do uso racional de } \\
\text { medicamentos entre adultos em } \\
\text { alfabetização na rede municipal } \\
\text { de ensino em Uberlância-MG }\end{array}$ & Ribeiro. S. C. & 2011 \\
\hline Revista Brasileira de Enfermagem & $\begin{array}{l}\text { Polifarmácia: interaçōes e reaçòes } \\
\text { adversas no uso de medicamentos } \\
\text { por idosos }\end{array}$ & Secoli, S. R. & 2010 \\
\hline Cadernos de Saúde Pública & $\begin{array}{l}\text { Utilização de medicamentos por } \\
\text { idosos brasileiros, de acordo com } \\
\text { a faixa etária: um inquérito postal }\end{array}$ & $\begin{array}{l}\text { Silva, A., Ribeiro, A., } \\
\text { Klein, C., \& Francisco, A. }\end{array}$ & 2012 \\
\hline $\begin{array}{l}\text { Brazilian Journal of Surgery and } \\
\text { Clinical Research }\end{array}$ & $\begin{array}{l}\text { Polifarmácia em idosos: } \\
\text { consequências de polimorbidades }\end{array}$ & $\begin{array}{l}\text { Tinôco, E., Costa, E. } \dot{E}_{.+} \\
\text {Sousa, K., Marques, M.. } \\
\text { Marques, T., Martins, V., } \\
\text { Júnior, A., \& Saliba, W. }\end{array}$ & 2021 \\
\hline $\begin{array}{l}\text { Revista Ciências do Ambiente On- } \\
\text { Line }\end{array}$ & $\begin{array}{l}\text { Impacto ambiental do descarte de } \\
\text { fármacos e estudo da } \\
\text { conscientização da população a } \\
\text { respeito do problema }\end{array}$ & $\begin{array}{l}\text { Ueda, J., Tavernaro, R., } \\
\text { Marostega, V., \& Pavan, } \\
\text { W. }\end{array}$ & 2009 \\
\hline $\begin{array}{l}\text { Revista de gestão em sistemas de } \\
\text { saúde }\end{array}$ & 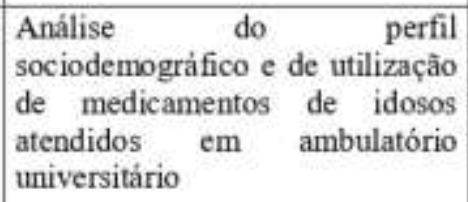 & $\begin{array}{l}\text { Vieiro, M., Perseguino, } \\
\text { M., Rastelli, V., Vismari, } \\
\text { L. }\end{array}$ & 2020 \\
\hline Santa Maria & 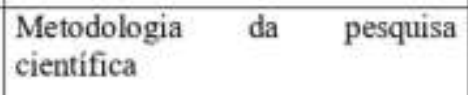 & Pereira, A.S. et al. & 2018 \\
\hline Einstein (São Paulo) & $\begin{array}{l}\text { Revisão integrativa: o que é e } \\
\text { como fazer }\end{array}$ & $\begin{array}{l}\text { Souza, M. T. D., Silva, M. } \\
\text { D. D., \& Carvalho, R. D. }\end{array}$ & 2010 \\
\hline Ciéncia \& Saúde Coletiva & $\begin{array}{l}\text { Uso de rastreadores para busca de } \\
\text { reaçỏes adversas a medicamentos } \\
\text { como motivo de admissão de } \\
\text { idosos em pronto-socorro }\end{array}$ & $\begin{array}{l}\text { Nagai, K. L., Takahashi, P. } \\
\text { S. K, Pinto, L. M. O., } \\
\text { Romano-Lieber, N. S. }\end{array}$ & 2018 \\
\hline Ciência \& Saúde Coletiva & $\begin{array}{l}\text { Uso de } \quad \begin{array}{r}\text { medicamentos } \\
\text { potencialmente inapropriados em }\end{array}\end{array}$ & $\begin{array}{l}\text { Moreira, F. S. M., Jerez- } \\
\text { Roig, J., Ferreira, L. M. B. } \\
\text { M., Dantas, A. P. Q. M.. }\end{array}$ & 2020 \\
\hline
\end{tabular}




\begin{tabular}{|l|l|l|l|}
\hline & $\begin{array}{l}\text { idosos institucionalizados: } \\
\text { prevalência e fatores associados }\end{array}$ & $\begin{array}{l}\text { Lima, C. K., Ferreira, M. } \\
\text { A. F. }\end{array}$ \\
\hline Ciência \& Saúde Coletiva & $\begin{array}{l}\text { Prescrição de medicamentos } \\
\text { potencialmente inapropriados } \\
\text { para idosos: um estudo na } \\
\text { Atençăo Primária à Saúde }\end{array}$ & $\begin{array}{l}\text { Farias, A. D., Lima, K. C., } \\
\text { Oliveira, Y. M. C., Leal, } \\
\text { A. A. F., Martins, R. R., } \\
\text { Freitas, C. H. S. M. }\end{array}$ & 2021 \\
\hline Revista Brasileira de Enfermagem & $\begin{array}{l}\text { Problemas relacionados a alente, S. H., Barbosa, S. } \\
\text { medicamentos na transição de } \\
\text { idosos do hospital para casa }\end{array}$ & $\begin{array}{l}\text { Val, Ferro, D., Fabriz, L. } \\
\text { A., Schonholzer, T. E., } \\
\text { Pinto, I. C. }\end{array}$ \\
\hline
\end{tabular}

Fonte: Autores.

\section{Resultados e Discussão}

A parcela de idosos na população brasileira vem crescendo muito nas últimas décadas. Devido às ações de saúde pública, sobretudo como vacinação, saneamento básico, os processos de urbanização e planeamento familiar, assim como também os avanços médico-tecnológicos, houve um grande aumento da expectativa de vida da população (Nóbrega \& Karnikowsky, 2005).

De acordo o Ministério da Saúde do Brasil, para a Organização Mundial da Saúde (OMS) a terceira idade é aquela composta por pessoas com mais de 65 anos, mas o Brasil segue o disposto pela Organização das Nações Unidas (ONU), a velhice tem início a partir dos 60 anos.

Conforme os dados do Instituto Brasileiro de Geografia e Estatística (IBGE), a população brasileira deverá atingir até 2042 cerca de 228,4 milhões de idosos. A expectativa é que o tempo de vida do brasileiro atinja os 80,0 anos em 2041 e 81,2 anos até 2060 (IBGE, 2013).

Durante o processo de envelhecimento manifestam-se limitações causadas pela idade. Surge a necessidade de implementação de terapias medicamentosas de alta ou baixa complexidade em decorrência de doenças agudas ou crônicas que aparecem ao decorrer do tempo e tendem a se intensificar na velhice (Silva et al., 2012).

A junção da presença de doenças, alterações fisiológicas que o corpo sofre, e atendimento médico variados, faz com que os idosos se tornem o principal grupo de risco para utilizar medicamentos de forma incorreta, o que acarreta vários riscos, como efeitos adversos, intoxicações, alergia e até mesmo retardo de diagnóstico de alguma doença. Ao avaliar que o cuidado com os idosos não advém de forma integral, ou seja, por um único especialista, o número de casos desses problemas e duplicidade de medicamentos são fartos e frequentes devido o atendimento realizado por diferentes médicos que não observam as prescrições já existentes para aquele idoso (Secoli, 2010). E por este motivo é tão importante assistir a ingestão medicamentosa em idosos.

Valente et al. (2019), relatam com base em dados do Ministério da Saúde que ocorrem em média entre 1,2 e 3,2 milhões de internações hospitalares de urgência de idosos por problemas relacionados a medicamentos por ano. Estes problemas podem gerar até 3,6 bilhões de reais de custos para o Sistema Único de Saúde (SUS) anualmente.

Segundo Oliveira (2013), a prática da polifarmácia por idosos gera a ocorrência de efeitos negativos e não intencionais nos mesmos, podendo estar relacionada à condição clínica do idoso e também com as características dos fármacos. A polifarmácia consiste no uso de mais de um medicamento para tratar diversas enfermidades, o que pode ter como consequência erros de medicação. Nos estudos de Secoli (2010), erros de medicação foram verificados em 15\% dos idosos que fazem uso de um medicamento, e $35 \%$ naqueles que fazem uso de mais de quatro medicamentos. Além disso, estima-se que $23 \%$ da população brasileira consome $60 \%$ daqueles medicamentos que estão disponíveis no mercado.

Para Tinôco et al. (2021), o uso de polifarmácia também aumenta as chances de interações medicamentosas e reações adversas. As interações medicamentosas são uma resposta farmacológica da interação de um medicamento ou substância com outro ingerido simultaneamente ou anteriormente ao primeiro. Quando estes fatores ocorrem os idosos podem ser acometidos a 
distúrbios gastrointestinais, alergias, tonturas, alterações nos ritmos cardíacos e respiratórios, etc, o que aumenta a procura pela rede de saúde por algo que poderia ser evitado.

Costa e Pedroso (2010) relatam que com o processo de envelhecimento o corpo passa a sofrer modificações farmacocinéticas relacionadas a absorção, distribuição, metabolismo e excreção, o que afeta diretamente a absorção de medicamentos, podendo causar aumento do pH gástrico e redução do fluxo sanguíneo do sistema digestório.

As interações medicamentosas são classificadas em graves, moderadas e leves. A interação medicamentosa grave, importante ou maior pode causar danos irreversíveis e motivar riscos à saúde dos idosos, a moderada pode ocasionar um regresso no tratamento ou necessidade de implantar outro tratamento concomitante por conta do aumento de um problema, e a leve, secundária ou menor acarreta apenas algum incômodo ao idoso. Há ainda a interação medicamentosa contraindicada, que ocorre quando foi feito o uso concomitante de medicamentos contraindicados (Garske et al., 2016).

No estudo de Gotardelo et al. (2014), os fármacos mais presentes em interações medicamentosas foram os antiinflamatórios e os medicamentos usados para tratar doenças cardiovasculares, dentre eles cite-se a interação entre anlodipino e diclofenaco, alodipino e ibuprofeno, metformina e nifedipino, ácido acetilsalicílico e hidroclorotiazida, anlodipino e sinvastatina, digoxina e hidroclorotiazida, losartana e enalapril.

Fora isto, as reações adversas também são consequências causadas pelo uso de medicamentos. Definidas como efeitos indesejáveis e prejudiciais causados pela administração desorganizada de medicamentos, causam riscos desnecessários aos idosos, como tonturas, quedas, desidratação e desequilíbrio iônico. Medicamentos psicóticos causam sedação, fraqueza e tremores, e os diuréticos, fadiga e desidratação (Tinôco et al., 2021).

Segundo Nagai, Takahashi, Pinto e Romano-Lieber (2018), as reações medicamentosas em pacientes internados podem interromper tratamentos, aumentar o tempo de internação e consequentemente as despesas hospitalares, caso esteja internado na rede particular de saúde. Além disso, as reações medicamentosas são quatro vezes mais comuns em idosos do que em não-idosos, em decorrência das alterações fisiológicas em virtude do envelhecimento e a quantidade de remédios ingeridos para tratar doenças crônicas. Em seus estudos ficou constatado que as suspeitas mais frequentes de reações medicamentosas em idosos partem de medicamentos da classe A, como os do trato alimentar e metabolismo, e os da classe C, relativos ao sistema cardiovascular.

Desta forma, a análise individualizada da prescrição e ação de cada medicamento utilizado por um idoso é muito importante, pois evita o acontecimento de interações medicamentosas e/ou reações adversas (Tinôco et al., 2021). Para Moreira et al. (2020), a relação risco-benefício de medicamentos deve ser levada em consideração, e por isto as listas de Medicamentos Potencialmente Inapropriados para Idosos, publicadas por vários autores, são tão importantes. Os medicamentos classificados como potencialmente inapropriados são aqueles que as chances de provocarem reações adversas são maiores que os benefícios anunciados. Em seus estudos concluiu-se que os medicamentos potencialmente inapropriados para idosos são os antipsicóticos, os benzodiazepínicos e os sulfonilureias, tendo incidência em $54,6 \%$ dos participantes do estudo e estão associados a prática da polifarmácia.

Aliado a polifarmácia, a automedicação também é um grande problema nesta faixa etária. Realizada tanto pelo próprio idoso, como por ordem de seu responsável, tem por objetivo obter melhoras no tratamento de uma enfermidade ou conforto de sintomas, e é feita sem a orientação de um profissional de saúde. Algumas das causas que contribuem para a automedicação de idosos são as experiências que deram certo anteriormente com o uso de algum medicamento, a familiaridade com classes medicamentosas, seja por indicação de amigos ou parentes, seja por uso em outras ocasiões, e os obstáculos para se ter um acompanhamento médico pelos serviços de saúde (Bortolon et al., 2008). 
Nos estudos de Farias et al. (2021), os idosos participantes informaram fazer uso de medicamentos além dos que foram prescritos, colaborando com a prática da automedicação, não sabendo informar sequer o nome dos medicamentos. Em estudo de âmbito nacional, observou-se que 14,3\% dos idosos praticam a automedicação.

De acordo Piotto (2009), os analgésicos e anti-inflamatórios são os fármacos mais encontrados na automedicação. E, ainda, que em muitas ocasiões, a prática da automedicação possa ser considerada uma necessidade ao sistema de saúde, é importante que medidas de orientação sobre o uso adequado de medicamentos sejam ensinadas. Um dos fatores que contribuem para a automedicação é a dificuldade de acesso ao sistema de saúde.

As deficiências presentes na saúde pública agravam ainda mais o problema da automedicação em idosos. Isto porque a demora em conseguir atendimento na rede pública de saúde e a comercialização de medicamentos de forma tão acessível pelos meios de comunicação contribuem para que o idoso se automedique (Bortolon et al., 2008).

As grandes falhas na inclusão de idosos, historicamente marginalizadas no Brasil, são evidenciadas na trajetória do SUS. As políticas públicas em saúde devem voltar-se a implantação da equidade, por meio da atenção específica aos idosos por necessitar de atenção peculiar e particularizada (Freitas et al., 2011).

Outro fator que colabora com a prática da automedicação é o estoque de medicamentos em casa, o que representa um risco de intoxicação para eles, além de induzir ao uso desnecessário devido a facilidade de acesso. $\mathrm{O}$ armazenamento inadequado influencia na garantia da qualidade do produto e pode decompor seu efeito, pois todo medicamento tem atributos físicos, químicos e condições microbiológicas especiais. A conservação de suas características e sua duração podem ser alteradas devido fatores como o tempo, apresentação de oxigênio, luz solar, radiação e umidade (Pereira et al., 2006).

O local ideal para armazenamento de medicamentos é aquele que possui circulação de ar, mas sem amparo da luz, calor e umidade, descartando ambientes como o banheiro e cozinha, de forma a dificultar a deterioração do produto (Oliveira et al., 2020).

Dias et al. (2009), destacam ainda a destreza decisiva e reflexiva de informações trazidas pelos meios de comunicação, e demostram sua inquietação quanto a prática e uso de caráter filantrópico pelos usuários dos meios de comunicação social.

A influência da mídia, mais especificamente das propagandas, é motivo de preocupação em alguns estudos. As propagadas publicitárias, que trazem consigo um vocabulário fácil e frases curtas, induzem o consumo de medicamentos com a promessa de alívio rápido e fazem com que a automedicação seja ainda a primeira alternativa para alívio de dores fortes (Junior et al., 2010). No mais, as propagandas se usam de artimanhas que tendem a direcionar as pessoas a adquirir produtos muitas vezes por questões comerciais, omitindo os riscos inerentes a sua utilização (Nascimento, 2010).

Ademais, o descarte incorreto de medicamentos gera problemas além do corpo-organismo humano. $\mathrm{O}$ descarte de medicamentos deve ser feito em local apropriado. Pouco se fala sobre isto na mídia, mas o descarte de medicamentos não deve ser feito no lixo comum, pois são elementos tóxicos e que podem contaminar o solo, água e animais que se alimentem de plantas deste solo e água (Ueda et al., 2009).

Ueda et al. (2009) informam que o descarte em local indevido de medicamentos vencidos pode acarretar estragos ao meio ambiente, como por exemplo, água contaminada por hormônio feminino pode afetar o sistema reprodutivo, o que motiva a feminização de peixes machos. As bactérias podem adquirir aversão a antibióticos em ambientes contaminados. Já no solo, os fármacos podem corromper os alimentos e, consequentemente, os animais que se alimentam do que brota da terra contaminada.

Com base no art. 59 da Resolução da Diretoria Colegiada (RDC) nº 222/18 da Agência Nacional de Vigilância Sanitária (ANVISA), que regulamenta as boas práticas de gerenciamento dos resíduos de serviços de saúde e outras providências, medicamentos que contenham produtos hormonais, antimicrobianos, imunossupressores, antineoplásicos, digitálicos, citostásticos, imunomoduladores e anti-retrovirais ao serem descartados por farmácias, drogarias e serviços assistenciais de saúde devem ser submetidos a tratamento e despejados em aterros de resíduos perigosos de classe I, que consiste em um local para 
disposição final de resíduos perigosos no solo, sem a causar riscos à saúde pública e impactos ambientais, pois usa recursos de engenharia de confinamento de resíduos.

Ueda et al. (2009) alertam para os prejuízos causados ao meio ambiente devido a contaminação da água e do solo por fármacos, pois estes não são eliminados no processo de tratamento de esgoto. $\mathrm{O}$ descarte de medicamentos em locais inapropriados é um problema de saúde pública. Cabe, mais uma vez, à educação exercer o papel de orientadora e alertar quanto as consequências trazidas ao meio ambiente.

De acordo a RDC n 222/18 da ANVISA, materiais provenientes de drogarias e farmácias são geradores de resíduos de serviços de saúde (RSS), pois estão relacionados a atenção a saúde humana. A coleta desses resíduos deve ser realizada de acordo horários e rota definidos dos centros de geração até o abrigo externo ou temporário, e em coletor de material liso, impermeável, rígido, lavável e com tampa vinculada ao corpo do coletor. Nos abrigos, os RSS devem ser mantidos em sacos acondicionados dentro do coletor com a tampa fechada e seguem para tratamento e/ou descarte em aterros de classe I.

Isto posto, a forma encontrada para minimizar ou ainda interromper os danos causados pelo uso inadequado de medicamentos é por meio da educação, da disseminação de informações e conhecimento.

Os medicamentos são elementos importantes na recuperação e promoção da saúde em circunstâncias diferentes, tanto que o seu costume toma caráter fundamental dentre as alternativas terapêuticas disponíveis e concebe destaque na economia global.

Desta maneira, torna-se importante não apenas aprovar a promoção, mas especialmente solicitar o uso coerente dos medicamentos e abonar a segurança no consumo, o que deve ser o enfoque dos organismos unidos ao sistema de saúde (Ribeiro, 2011).

É fundamental destacar que a terapia medicamentosa é somente uma das frentes de intervenção para alívio de sintomas de doenças. A Organização Mundial de Saúde (OMS) dita que a saúde não se trata apenas da ausência de doença ou enfermidade, mas deve compreender também além do bem-estar físico, o completo bem-estar mental e social.

A observação cautelosa de fatores que envolvem o uso de medicamentos, como dose, horários e a orientação adequada dos idosos e seus familiares, são alguns dos elementos essenciais para ajudar a manter uma boa qualidade de vida para o idoso. Sabe-se que o uso de medicamentos em qualquer idade pode acarretar reações indesejadas, porém a incidência aumenta proporcionalmente com a idade.

É importante também que haja humanidade por parte de médicos, enfermeiros e farmacêuticos na hora da prescrição de remédios a idosos. A explicação deve ser calma e com clareza, e muito das vezes repetitiva, mas tudo em prol de fazer com que o idoso entenda perfeitamente o que precisa ser feito.

\section{Conclusão}

Uma vez que o processo de humanização está entrelaçado com a educação, as mudanças comportamentais tornam-se inerentes. A educação conduz o idoso da ignorância ao conhecimento, da dependência à autonomia, da inconsciência à consciência de si mesmo e sua função na sociedade. É essencial que os farmacêuticos, unidos aos médicos junto à comunidade, despertem para este processo e se atrelem de forma interdisciplinar para que possam contribuir na formação da cidadania e autonomia de idosos.

Restou constatado por meio desta pesquisa que é recorrente entre idosos a prática da automedicação, a polifarmácia e o descarte de medicamentos em locais indevidos, resultantes do atendimento do idoso por diferentes médicos com especialidades diferentes que muitas vezes não levam em consideração prescrições anteriores, as deficiências existentes no sistema público de saúde e a comercialização acessível e de fácil entendimento de medicamentos na mídia. Conclui-se que a prática da polifarmácia 
e da automedicação são causadoras de interações medicamentosas entre a orgânica de fármacos e reações adversas indesejadas, além de que o descarte incorreto de medicamentos em locais indevidos pode causar desequilíbrios no meio ambiente.

Considera-se, portanto, importante que futuramente sejam realizadas pesquisas e trabalhos para avaliar a chegada de novos medicamentos potencialmente inapropriados no mercado, a interação entre eles no organismo do idoso, se houve aumento ou estabilidade das práticas da polifarmácia e automedicação por idosos, se a consciência do descarte de medicamentos em locais corretos virou hábito entre esta faixa etária, e nas demais também.

\section{Referências}

Bortolon, P., Medeiros, E., Naves, J., Karnikowsky, M., \& Nóbrega, O. (2008). Análise do perfil de automedicação em mulheres idosas brasileiras. Ciência \& Saúde Coletiva, 13(4), 1219-1226.

Costa, S. C. C., \& Pedroso, E. R. P. (2010). A prescrição de medicamentos para idosos internados em serviço de clínica médica: atualização.

Dias, F., Silva, K., Lima, F., \& Pinheiro, P. (2009). A influência da mídia na promoção da Saúde. In: Congresso Brasileiro de Enfermagem, 61º, trabalho 182, $159-162$.

Farias, A. D., Lima, K. C., Oliveira, Y. M. C., Leal, A. A. F., Martins, R. R., Freitas, C. H. S. M. (2021). Prescrição de medicamentos potencialmente inapropriados para idosos: um estudo na Atenção Primária à Saúde. Ciência \& Saúde Coletiva, 26(05).

Flores, L. M., \& Mengue, S. S. (2005). Uso de medicamentos por idosos em região sul do Brasil. Revista Saúde Pública.

Freitas, D., Caballero, A., Marques, A., Hernández, C., \& Antunes, S. (2011). Saúde e comunidades quilombolas: uma revisão da literatura. Rev. CEFAC, 13(5), 937-943.

Garske, C., Assis, M., Schneider, A., Machado, E., \& Morsch, L. (2016). Interações medicamentosas potenciais na farmacoterapia de idosos atendidos em farmácia básica do sul do Brasil. Santa Maria, 42(2), 97-105.

Gotardelo, D., Fonseca, L., Masson, E., Lopes, L., Toledo, V., Faioli, M., Meira, A., Costa, C., \& Andrade, R. (2014). Prevalência e fatores associados a potenciais interações medicamentosas entre idosos em um estudo de base populacional. Rev. Bras. Med. Fam. Comunidade, 9(31), 111-118.

IBGE. (2018). Número de idosos cresce 18\% em 5 anos e ultrapassa 30 milhões em 2017. https://agenciadenoticias.ibge.gov.br/agencia-noticias/2012-agenciade-noticias/noticias/20980-numero-de-idosos-cresce-18-em-5-anos-e-ultrapassa-30-milhoes-em-2017

IBGE. (2013). População brasileira deve chegar ao máximo 228,4 milhões em 2042. https://agenciadenoticias.ibge.gov.br/agencia-sala-de-imprensa/2013agencia-de-noticias/releases/14462-asi-populacao-brasileira-deve-chegar-ao-maximo-2284-milhoes-em-2042

Junior, D., Neves, A., Cerqueira, C., Marcellini, P., Marques, T., \& Barros, J. (2010). Influência da propaganda na utilização de medicamentos em um grupo de idosos atendidos em uma unidade básica de saúde em Aracajú (SE, BRASIL). Ciência \& Saúde Coletiva, 15(3), $3497-3505$.

Melo, L. M., \& Vale, B. N. (2018). Polifarmácia: uso de medicamentos por idosos. Revista Amazônica Science \& Health.

Ministério da Saúde. Saúde da pessoa idosa: prevenção e promoção à saúde integral. https://antigo.saude.gov.br/saude-de-a-z/saude-da-pessoa-idosa

Moreira, F. S. M., Jerez-Roig, J., Ferreira, L. M. B. M., Dantas, A. P. Q. M., Lima, C. K., \& Ferreira, M. A. F. (2020). Uso de medicamentos potencialmente inapropriados em idosos institucionalizados: prevalência e fatores associados. Ciência \& Saúde Coletiva, (25)06.

Nagai, K. L., Takahashi, P. S. K, Pinto, L. M. O., \& Romano-Lieber, N. S. (2018). Uso de rastreadores para busca de reações adversas a medicamentos como motivo de admissão de idosos em pronto-socorro. Ciência \& Saúde Coletiva, (23)11.

Nascimento, À. C. (2010). Propaganda de medicamentos para grande público: parâmetros conceituais de uma prática produtora de risco. Rio de Janeiro: Ciência \& Saúde.

Nóbrega, O. T., \& Karnikowsky, M. G. O. (2005). A terapia medicamentosa no idoso: cuidados na medicação. Distrito Federal: Ciência \& Saúde Coletiva.

Oliveira, A. M. (2018). Fatores de risco associados à polifarmácia no idoso. Minas Gerais: Universidade Aberta do SUS.

Oliveira, D., Jesus, P., Zucco, B., Panosso, É., Rocha, V., Bayer, V., \& Ries, E. (2020). Desenvolvimento, validação e utilização de material educativo sobre armazenamento correto de medicamentos. Saúde e Pesquisa, 13(3), 461-473.

Oliveira, M. P. F., \& Novaes, M. R. C. G. (2012). Uso de medicamentos por idosos de instituições de longa permanência, Brasília-DF, Brasil. Distrito Federal: Revista Brasileira de Enfermagem.

Pereira, A.S. et al. (2018). Metodologia da pesquisa científica. UFSM.

Pereira, J., Soares, L., Hoepfner, L., Kruger, K., Guttervil, M., Tonini, K., Devegili, D., Rocha, E., Verdi, F., Dalfovo, V., Oslen, K., Mendes, T., Deretti, R., Soares, V., Lobermeyer, C., Moreira, J., Ferreira, J., \& Francisco, A. (2006). Riscos da automedicação: tratando o problema com conhecimento. UNIVILLE.

Piotto, F., Nogueira, R., Pires, O., et al. (2009). Prevalência da dor e do uso de analgésicos e antiinflamatórios na automedicação de pacientes atendidos no Pronto-Socorro Municipal de Taubaté. Revista Dor, 10(4), 313-7. 
Research, Society and Development, v. 10, n. 15, e560101522950, 2021

(CC BY 4.0) | ISSN 2525-3409 | DOI: http://dx.doi.org/10.33448/rsd-v10i15.22950

Resolução da Diretoria Colegiada n 222, de 28 de março de 2018. Regulamenta as Boas Práticas de Gerenciamento dos Resíduos de Saúde e dá outras providências. Brasília, DF. https://bvsms.saude.gov.br/bvs/saudelegis/anvisa/2018/rdc0222_28_03_2018.pdf

Ribeiro, S. C. (2011). Educação em saúde para a promoção do uso racional de medicamentos entre adultos em alfabetização na rede municipal de ensino em Uberlância-MG. Dissertação. Distrito Federal: Universidade de Brasília.

Secoli, S. R. (2010). Polifarmácia: interações e reações adversas no uso de medicamentos por idosos. Revista Brasileira de Enfermagem.

Silva, A., Ribeiro, A., Klein, C., \& Francisco, A. (2012). Utilização de medicamentos por idosos brasileiros, de acordo com a faixa etária: um inquérito postal. Cadernos de Saúde Pública, 28(6), 1-13.

Souza, M. T. D., Silva, M. D. D., \& Carvalho, R. D. (2010). Revisão integrativa: o que é e como fazer. Einstein (São Paulo), 8(1), $102-106$.

Tinôco, E., Costa, É., Sousa, K., Marques, M., Marques, T., Martins, V., Júnior, A., \& Saliba, W. (2021). Polifarmácia em idosos: consequências de polimorbidades. Brazilian Journal of Surgery and Clinical Research, 35(2), 79-85.

Ueda, J., Tavernaro, R., Marostega, V., \& Pavan, W. (2009). Impacto ambiental do descarte de fármacos e estudo da conscientização da população a respeito do problema. Revista Ciências do Ambiente On-Line, 5(1).

Valente, S. H., Barbosa, S. M., Ferro, D., Fabriz, L. A., Schonholzer, T. E., \& Pinto, I. C. (2019). Problemas relacionados a medicamentos na transição de idosos do hospital para casa. Revista Brasileira de Enfermagem, 72(02).

Vieiro, M., Perseguino, M., Rastelli, V., \& Vismari, L. (2020). Análise do perfil sociodemográfico e de utilização de medicamentos de idosos atendidos em ambulatório universitário. Revista de gestão em sistemas de saúde, 9(3). 